\title{
142. Conversion of Somatic Antigen 20 and Transduction of Genetic Characters by Bacteriophage in Salmonella Group C
}

\author{
By Shoei Iseki and Toshio Matsumoto \\ Department of Legal Medicine, School of Medicine, \\ Gunma University, Maebashi, Japan \\ (Comm. by T. Furuhata, M.J.A., Dec. 12, 1959)
}

Since Iseki and Sakai ${ }^{1}$ discovered conversion by bacteriophage in Salmonella group $\mathrm{E}$ with regard to somatic antigen 15 , similar phenomena have been found one after another up to the present. Iseki and Kashiwagi ${ }^{233)}$ reported on antigenic conversion by bacteriophage with regard to somatic antigen 1 of Salmonella groups A, B, and D, $\mathrm{Harada}^{4)}$ reported the same with regard to somatic antigen 34 of Salmonella group E, Baron, Formal, and Washington ${ }^{5)}$ with regard to somatic antigen 20 of Salmonella group C, Matsui ${ }^{6}$ ) with regard to type antigen IV, that is, $\mathrm{IV}_{1}$ by Iseki and Hamano, ${ }^{7)}$ of Shigella flexneri.

On the other hand, Zinder and Lederberg ${ }^{8)}$ observed in Salmonella typhi murium the fact that genetic characters could be transferred from one strain to another, and they called this phenomenon "transduction". Now it is known that the phage which participates in the conversion of the somatic antigen of Salmonella has the ability to transduce the genetic characters. Iseki and Sakai ${ }^{9}$ reported on epsilon 15 phage, Iseki and Kashiwagi ${ }^{10)}$ on iota phage, and Iseki and Hamano $^{11)}$ on epsilon 34 phage, all of which have the ability to induce not only conversion of somatic antigens 15,1 , and 34 , respectively, but also the transduction of the genetic characters.

Now we have confirmed the conversion of somatic antigen 20, induced by the bacteriophage from Salmonella group C as reported by Baron, Formal, and Washington,"5) and elucidated the relationship between this bacteriophage and other temperate phage, and further found that this in addition possesses the ability of the transduction similar to bacteriophage, reported by Spilman, Baron, and Formal, ${ }^{12}$ which is derived from a Salmonella group C strain having somatic antigens 6,7 .

Materials and methods. 1. Strains. The strains of Salmonella group C, employed in the experiments are listed in Table I.

2. Investigation for lysogenicity and sensitivity. The bacterial liquid was spread on the agar plate, dried, and then culture filtrate of the bacterium was dropped on it, and after 12 hours incubation, the plate was investigated for lytic plaques. At the same time, enu- 
Table I. Strains of Salmonella group C used in the experiments

\begin{tabular}{l|c|l|c}
\hline \multicolumn{1}{c|}{ Nomenclature } & Abbreviation & Antigenic structure & $\begin{array}{c}\text { Inositol-ferment- } \\
\text { ing ability }\end{array}$ \\
\hline $\begin{array}{l}\text { S. newport var. } \\
\text { puertorico }\end{array}$ & $\mathrm{S}-51$ & $6,8:-: 1,2,3$ & - \\
S. muenchen & $\mathrm{S}-54$ & $6,8: \mathrm{d}: 1,2$ & + \\
S. narashino & $\mathrm{S}-55$ & $6,8: \mathrm{a}: \mathrm{e}, \mathrm{n}, \mathrm{x}$ & + \\
S. duesseldorf & KC-121 & $6,8: \mathrm{Z}_{4}, \mathrm{Z}_{24}:-$ & + \\
S. emek & KCIII-3 & $(8), 20: \mathrm{g}, \mathrm{m}, \mathrm{s:-}$ & + \\
S. corvallis & KCIII-5 & $(8), 20: \mathrm{Z}_{4}, \mathrm{Z}_{23}:-$ & + \\
S. albany & KCIII-6 & $(8), 20: \mathrm{Z}_{4}, \mathrm{Z}_{24}:-$ & + \\
S. kentucky 21883 & & $(8), 20: \mathrm{i}: \mathrm{Z}_{6}$ & + \\
\hline
\end{tabular}

meration of lytic plaques was made by the agar layer method using $0.8 \%$ semisolid agar. The bacterium, in the culture filtrate of which a phage was found to be present, was classified as the lysogenic strain, and those which were lysed by such phage, as the sensitive strain.

3. Factor serum. To prepare anti-20 and anti-6 factor sera, rabbits were immunized with $S$. albany ((8), 20: $\left.\mathrm{Z}_{4}, \mathrm{Z}_{24}:-\right)$ and $S$. newport var. puertorico $(6,8:-: 1,2,3)$, both after heating at $100^{\circ} \mathrm{C}$ for 2 hours, and the obtained antisera were reciprocally absorbed with each other. Anti-phage serum was prepared by immunizing rabbits with phage liquid.

4. Experiments on conversion of somatic antigen 20. To 1 cc of broth, containing $0.2 \mathrm{cc}$ of culture filtrate of strain with somatic antigens (8), 20, was added a small amount of bacteria with somatic antigens 6, 8, and the whole was incubated for 24 hours. A portion of it was then spread on the agar plate to yield bacterial colonies, each of which was investigated for agglutination with anti-20 and anti-6 factor sera.

5. Experiments on the transduction of the genetic characters. The phage liquid which was increased with streptomycin-resistant and phage-sensitive strain up to $10^{8} / \mathrm{cc}$ phage titer was allowed to act on streptomycin-sensitive and phage-sensitive strains, and it was investigated whether the streptomycin-resistance was transduced into the latter. Further, the phage liquid was increased with the strain which has inositol-fermenting ability as well as streptomycin-resistance, and this was allowed to act on the strain without such characteristics in order to investigate for the transduction of these.

Results. 1. Conversion by phage about somatic antigen 20 . When the culture filtrate of the strain with somatic antigens (8), 20 was allowed to act on the strain with somatic antigens 6 , 8, it was revealed, as shown by Table II, that the former contained the phage 
which could lyse S-51 and S-55, both of which possess somatic antigens 6 , 8, and that through lysogenization the strain without 20 was converted into a strain with somatic antigen 20 . Now the new strain possessed somatic antigens $6,8,20$, and so it had not lost the original antigen 6 , but newly acquired 20 in addition, as reported by Baron, Formal, and Washington. ${ }^{5)}$ In this way this conversion resembles that reported by Iseki and Kashiwagi ${ }^{2}{ }^{2}$ which takes place with regard to somatic antigen 1. Among strains with somatic antigens (8), 20, S. albany gave lower rate of conversion, and among strains with somatic antigens $6,8, \mathrm{~S}-54$ and $\mathrm{KC}-121$ were difficult to be lysed or lysogenized.

Table II. Conversion by phage about somatic antigen 20

\begin{tabular}{l|rrrr}
$\begin{array}{c}\text { Culture } \\
\text { filtrate }\end{array}$ & \multicolumn{4}{|c}{ Lysogenization and conversion } \\
& S-51 & S-55 & S-54 & KC-121 \\
S. kentucky & $11 / 100$ & $15 / 100$ & - & - \\
S. corvallis & $18 / 100$ & $12 / 100$ & - & - \\
S. emek & $20 / 100$ & $14 / 100$ & - & - \\
S. albany & $3 / 100$ & $1 / 100$ & - & -
\end{tabular}

The numerators denote the number of colonies per 100 in which antigenic conversion took place.

2. Properties of temperate phage. The phage derived from $S$. kentucky, acting on S-51, produced, as represented in Fig. 1, two kinds of lytic plaque: One was circular with a diameter of 2-3 mm, and the transparent central part was surrounded by a rather wide zone of turbidity, and around this there was a rather narrow zone of transparency; and the phage which produced this kind of lytic plaque was designated as Plt 20. The other was transparent and circular with a diameter of about $1 \mathrm{~mm}$, and the phage which produced this was designated as Psc. S. kentucky produced Plt 20 and Psc in the ratio of about 1:10. When infected with Plt 20, S-51 was lysogenized, at the same time acquiring somatic antigen 20, but when infected with Psc, S-51 was lysogenized, but without antigenic conversion. It was possible to infect S-51 simultaneously with these two kinds of phage to produce a doubly lysogenic strain. In neutralization experiments with anti-phage serum, Plt 20 could be neutralized only by anti-Plt 20 serum, and Psc only by anti-Psc serum. It is therefore known that these two kinds of phage have different antigenic characters from each other. Both lost their activities when Plt 20 was kept below $\mathrm{pH} 2.3$ and above $\mathrm{pH}$ 13, and Psc below $\mathrm{pH} 3.0$ and above $\mathrm{pH} 12.5$, in both cases at $5^{\circ} \mathrm{C}$ for 24 hours. They also lost activities to a degree on heating, the Plt 20 at $85^{\circ} \mathrm{C}$ for 30 minutes, and Psc mostly at $70^{\circ} \mathrm{C}$ for 10 minutes, and entirely at $80^{\circ} \mathrm{C}$ for 10 minutes. 
Through the electron microscope, Plt 20 was seen as particle with a diameter of about $70-80 \mathrm{~m} \mu$, and Psc with a diameter of 100-110 $\mathrm{m} \mu$ (Figs. 3 and 4). When Plt 20 was increased with S-51, a phage which has relatively clear lytic plaque was produced at the rate of 1 per 500, but this phage was similar to Plt 20 in the size of lytic plaque, and in the antigenicity, thermo-resistance, and $\mathrm{pH}$ range for its activity. This phage mutant was designated as Plc 20 (Fig. 2).

As above-mentioned, S. kentucky produced two kinds of phage, Plt 20 and Psc, and also S. corvallis released two similar kinds. But S. emek and S. albany each produced only one kind of phage with properties similar to those of Plt 20.

3. Experiments on the transduction of the genetic characters.

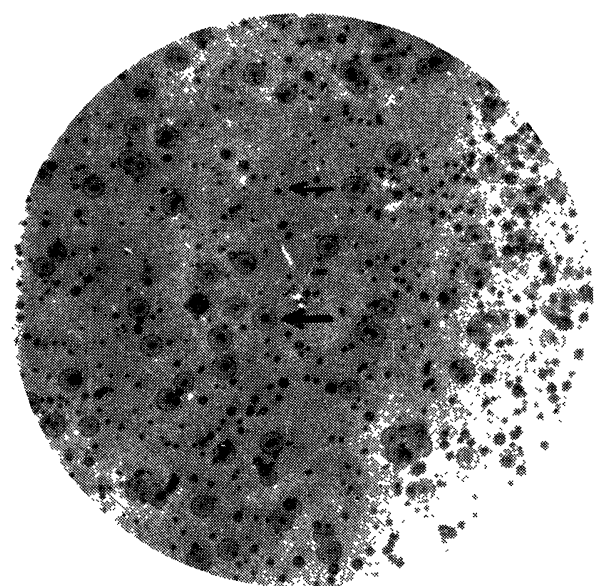

Fig. 1. Lytic plaques produced on $\mathrm{S}-51$ by the phages from S. kentucky. A thick arrow indicates a lytic plaque produced by Plt 20 , and a thin arrow indicates one produced by Psc.

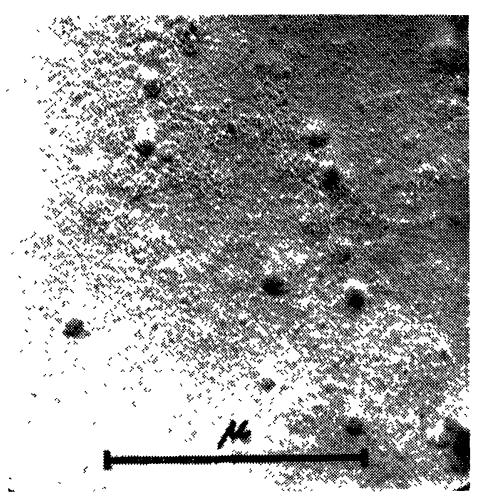

Fig. 3. Plt 20 magnified by the electron microscope. $\times 26,000$

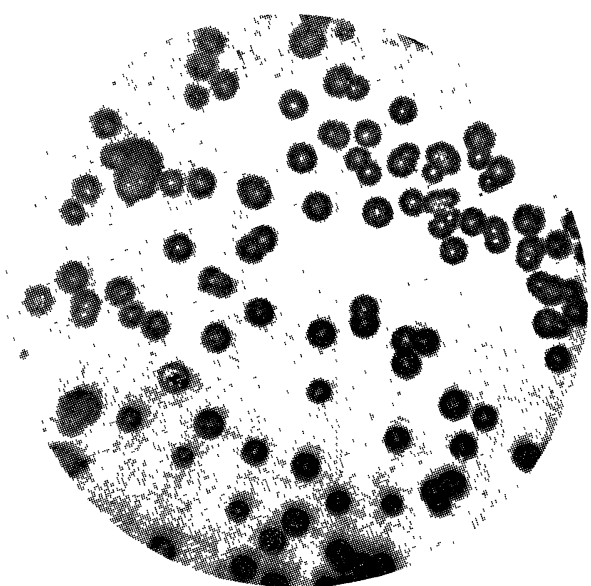

Fig. 2. Lytic plaques produced on S-51 by Plc 20 , mutant of Plt 20

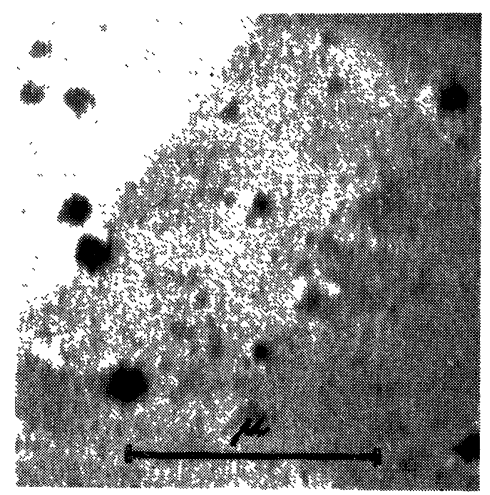

Fig. 4. Psc magnified by the electron microscope. $\times 26,000$ 
Plt 20 and its mutant Plc 20, and Psc were increased with streptomycin-resistant S-51, and with these, experiments were performed on the transduction of the streptomycin-resistance. The results were that in S-51 and S-55, which could easily be lysed or lysogenized by each of the above-mentioned phages, the transduction was induced by Plt 20 or Plc 20 at the rate of $10^{-6}-10^{-7}$. However, the transduction could not be observed in S-54 and $\mathrm{KC}-121$, which are difficult to be lysed or lysogenized with the phage and in strains with somatic antigens (8), 20. Psc phage, which has not the ability to induce antigenic conversion, could not induce the transduction of the character either.

In general, strains in which the streptomycin-resistance could be transduced were lysogenized, acquiring at the same time somatic antigen 20. When, however, Plt 20 from S. albany was used, there were organisms at the rate of 2-6 per 100 which were not lysogenized, nor acquired somatic antigen 20 , but in which nevertheless the transduction was performed. Thus it was shown that there are instances in which the transduction can take place without lysogenization.

Next, each phage was increased with S-55, which is streptomycinresistant and inositol-fermenting, and with these was infected S-51, which is streptomycin-sensitive and inositol non-fermenting, to investigate for the transduction. The results were that when Plt 20 and its mutant Plc were applied, the presence of organisms with the inositol-fermenting ability was confirmed by means of inositol-added peptone medium at the rate of $10^{-6}$, but not with inositol-added peptone medium which contained streptomycin, indicating that the transduction of both of the inositol-fermenting ability and streptomycin-resistance into one bacterial cell was difficult to take place. In general, bacteria into which inositol-fermenting ability was transduced were lysogenized, and acquired somatic antigen 20. And Psc, which had no ability to induce antigenic conversion, could not perform the transduction of the inositol-fermenting ability either.

Summary. Strains of Salmonella group C which have somatic antigens (8), 20 are lysogenic, and S. kentucky and S. corvallis, which are such lysogenic strains, produce two kinds of phage-Plt 20, which forms large turbid lytic plaques, and Psc, which forms small transparent lytic plaques. S. emek and S. albany, which are also lysogenic, produce only Plt 20. Against these, S. newport and S. narashino, which have somatic antigens 6,8 , are sensitive strains. When Plt 20 and its plaque mutant Plc 20 infect and lysogenize the sensitive strains, the somatic antigens 6,8 are converted into $6,8,20$, as reported by Baron, Formal, and Washington. ${ }^{5)}$ But when the lysogenization is induced by Psc, such conversion with regard to somatic antigen 20 
does not take place. Further, Plt 20 and its mutant Plc 20 possess, in addition to the above-mentioned ability of antigenic conversion, the ability to transduce such characters as streptomycin-resistance and inositol-fermentation into the sensitive strain at the rate of $10^{-6}-10^{-7}$. However, into one bacterial cell can generally be transduced only either one of the above two characters, but not both. Neither can Psc, which has no ability of antigenic conversion, induce the transduction of the genetic character.

\section{References}

1) Iseki, S., and Sakai, T.,: Artificial transformation of O antigens in Salmonella $\mathrm{E}$ group. II. Antigen-transforming factor in bacilli of subgroup $\mathrm{E}_{2}$, Proc. Japan Acad., 29 (3), 127-131 (1953).

2) Iseki, S., and Kashiwagi, K.,: Induction of somatic antigen 1 by bacteriophage in Salmonella B group, Proc. Japan Acad., 31 (8), 558-563 (1955).

3) Iseki, S., and Kashiwagi, K.,: Lysogenic conversion and transduction of genetic characters by temperate phage iota in Salmonella. I. Lysogenic conversion with regard to somatic antigen 1 in Salmonella groups A, B, and D, Proc. Japan Acad., 33 (8), 481-485 (1957).

4) Harada, K.,: Studies on directed variation of Salmonella 0-15 and 0-34 by bacteriophage. (1) Antigen transformation by two kinds of bacteriophage (in Jap.), Virus, 6 (4), 285-299 (1956).

5) Baron, L. S., Formal, S. B., and Washington, O.,: Somatic antigen addition in Salmonella by bacteriophage, Virology, 3 (2), 417-425 (1957).

6) Matsui, S.,: Antigenic changes in Shigella flexneri group by bacteriophage, Jap. J. Microb., 2 (2), 153-158 (1958).

7) Iseki, S., and Hamano, S.,: Conversion of type antigen IV in Shigella flexneri by bacteriophage, Proc. Japan Acad., 35 (7), 407-412 (1959).

8) Zinder, N. D., and Lederberg, J.,: Genetic exchange in Salmonella, J. Bact., 64 (5), 679-699 (1952).

9) Iseki, S., and Sakai, T.,: Transduction of biochemical properties in Salmonella E group, Proc. Japan Acad., 30 (2), 143-147 (1954).

10) Iseki, S., and Kashiwagi, K.,: Lysogenic conversion and transduction of genetic characters by temperate phage iota in Salmonella. II. Transduction of genetic characters by phage iota in Salmonella groups A, B, and D, Proc. Japan Acad., 33 (8), 486-491 (1957).

11) Iseki, S., and Hamano, S.,: The transduction and lysogenic conversion in Salmonella group E by means of epsilon 34 phage, Gunma J. Med. Sci., 7 (1), 1-6 (1958).

12) Spilman, W., Baron, L. S., and Formal, S. B.,: Correlation of phage action with the antigens of group C Salmonella, Bact. Proc., 51 (1955). 